\title{
Infection in Burns
}

\author{
William Norbury, David N. Herndon,, ${ }^{1,2}$ Jessica Tanksley, ${ }^{1,2}$ Marc G. Jeschke, ${ }^{3}$ and Celeste C. Finnerty ${ }^{1,2,4}$ \\ on Behalf of the Scientific Study Committee of the Surgical Infection Society
}

\section{Abstract}

Background: Developments in critical care and surgical approaches to treating burn wounds, together with newer antimicrobial treatments, have significantly reduced the morbidity and mortality rates associated with this injury.

Methods: Review of the pertinent English-language literature.

Results: Several resistant organisms have emerged as the maleficent cause of invasive infection in burn patients, including methicillin-resistant Staphylococcus aureus, vancomycin-resistant Enterococcus, Pseudomonas, Acinetobacter, non-albicans Candida spp., and Aspergillus. Advances in antimicrobial therapies and the release of new classes of antibiotics have certainly added to the armamentarium of therapeutic resources for the clinician.

Conclusion: Strict infection control measures, constant wound surveillance with regular sampling of tissues for quantitative culture, and early excision and wound closure remain the principal adjuncts to control of invasive infections in burn patients.

I NVASIVE INFECTION is now the chief reason for death and morbidity after burn injury, with it being responsible for $51 \%$ of the deaths $[1,2]$. The importance of prevention, surveillance, and sampling for infections in this immunocompromised group has been well established; however, there is a dearth of standard-of-care guidelines and novel approaches. In this review, we discuss the epidemiology of burn infections, define degrees of severity of infection, outline the major organisms associated with burn wounds, and describe the specific organs and tissues susceptible to infection. We also discuss strategies for surveillance, sampling, and infection control in the burn intensive care unit (ICU).

\section{Epidemiology}

Every year, approximately half a million Americans sustain burn injuries requiring medical intervention. Most of these do not require admission to a hospital. However, around 40,000 of these people are admitted, with $75 \%$ of them needing specialized treatment at a certified burn center. Advances in modern medical care incorporating aggressive fluid resuscitation, alleviation of the hypermetabolic response, adequate and effective surgical grafting and coverage of burn wounds, pulmonary toilet and ventilation, nutritional support, and infection control measures have ensured that the majority of patients reaching the hospital will survive. The total annual number of burn-related deaths is approximately 3,400 [3]. Sepsis and the accompanying invasive infection continue to be the primary reason for death after the first $24 \mathrm{~h}$, with these often culminating in the demise of the patient after the first 2 wks of admission. Over the last 10 years, the most frequent clinical complications reported in patients admitted to a certified burn center were pneumonia $(3.5 \%)$, cellulitis $(3 \%)$, and urinary tract infection $(2.6 \%)$. The frequency of pneumonia was greater in patients who had been injured by fire and those with four or more days of mechanical ventilation [4].

\section{Classification}

Burn wound impetigo, also referred to as graft ghostings and folliculitis when the scalp is involved, usually is caused by bacterial colonization rather than invasive

\footnotetext{
${ }^{1}$ Shriners Hospitals for Children, Galveston, Texas.

${ }^{2}$ Department of Surgery, ${ }^{4}$ Institute for Translational Science and Sealy Center for Molecular Medicine, University of Texas Medical Branch, Galveston, Texas.

${ }^{3}$ Ross Tilley Burn Centre, Sunnybrook Health Sciences Centre, Sunnybrook Research Institute, Department of Surgery, Division of Plastic Surgery and Department of Immunology, University of Toronto, Toronto, Ontario, Canada.
} 
infection. The result is a loss of epithelium from an area that had re-epithelialized.

Burn wound colonization may be diagnosed when bacteria are present at low concentrations $\left(<10^{5}\right.$ colony-forming units [CFU]) on the wound's surface. No surrounding erythema or cellulitis is evident, but deterioration of the wound surface can be observed.

Infection is defined as the presence of high concentrations ( $>10^{5}$ organisms/g of tissue) of bacteria in the burn wound and scab. However, no evidence exists that the infection is invasive. The presence of cellulitis is the foundation of the clinical diagnosis. Burn wound cellulitis can be observed when the area of erythema extends beyond what would be expected for the injury alone. It is the result of infection of the otherwise-healthy tissues surrounding the wound and often is accompanied by increased warmth within the area, pain or tenderness, advancing swelling, or induration. Occasionally, lymphangitis may be observed, but this is less common.

Invasive infection of burn wounds is a surgical emergency because of the high concentrations of bacteria $\left(>10^{5}\right.$ $\mathrm{CFU}$ ) in the wound and surrounding area, together with new areas of necrosis in unburned tissues. This situation often is accompanied by signs of sepsis and changes in the burn wound such as black, blue, or brown discoloration of the eschar. Urgent resuscitation measures are required, along with broad-spectrum antimicrobial agents, antifungals, and surgical debridement of the affected area. Specimens of this tissue must undergo histopathologic and microbiologic analysis to assist in the identification of the causative organism(s). Those patients with a delay in presentation or removal of burned tissues are at greatest risk of this condition [5].

Unfortunately, because of the natural pathophysiology of burns, definitions developed by the American College of Chest Physicians and Society of Critical Care Medicine for inflammatory response syndrome (SIRS), sepsis, severe sepsis, and septic shock inadequately describe the septic responses of the burn patient. Large burns, those covering $\geq 20 \%$ of the total body surface area, compromise the barrier function of the skin that prevents entry of microorganisms, triggering a prolonged, constant exposure to host immune response mediators that is not observed in other critical illnesses. The profound biphasic immune response, described as hyperinflammation followed by severe immunodepression, has a clinical presentation that mimics SIRS, sepsis, and the already-mentioned states. This finding led to the formation of a consensus conference in 2007 by members of the American Burn Association, who determined the definition of sepsis for burn patients that is used today [1].The group also adjudicated that the terms and clinical descriptions of SIRS, severe sepsis, and septic shock were not useful for the burn patient population. The definition of sepsis developed by the American Burn Association Consensus Conference to Define Sepsis and Infection in Burns is provided [1].

Sepsis is a change in the burn patient that triggers the concern for infection. It is a presumptive diagnosis where antibiotics are usually started, and a search for a cause of infection should be initiated. Whereas there is need for clinical interpretation, the diagnosis needs to be tied to the discovery of an infection. The definition is age-dependent with adjustments necessary for children.
The trigger includes at least three of the following:

I. Temperature $>39^{\circ} \mathrm{C}$ or $<36.5^{\circ} \mathrm{C}$

II. Progressive tachycardia
A. Adults $>110$ beats/min (bpm)
B. Children $>2$ SD [standard deviations] above age- specific norm $(85 \%$ age-adjusted maximum heart rate)
III. Progressive tachypnea

A. Adults $>25 \mathrm{bpm}$ if not ventilated i. Minute ventilation $>12 \mathrm{~L} / \mathrm{min}$ when ventilated

B. Children $>2$ SD above age-specific norm $(85 \%$ age-adjusted maximum respiratory rate)

IV. Thrombocytopenia (will not apply until $3 \mathrm{~d}$ after initial resuscitation)

A. Adults $<100,000 / \mathrm{mcL}$

B. Children $<2$ SD below age-specific norm

V. Hyperglycemia (in the absence of pre-existing diabetes mellitus)

A. Untreated plasma glucose $>200 \mathrm{mg} / \mathrm{dL}$ or equivalent $\mathrm{mM} / \mathrm{L}$

B. Insulin resistance-examples include

i. $>7$ units of insulin/h intravenous drip (adults)

ii. Resistance to insulin $(>25 \%$ increase in insulin requirements over $24 \mathrm{~h}$ )

VI. Inability to continue enteral feedings $>24 \mathrm{~h}$

A. Abdominal distension

B. Enteral feeding intolerance (residual $>150 \mathrm{~mL} / \mathrm{h}$ in children or $2 \times$ feeding rate in adults)

C. Uncontrollable diarrhea $(>2,500 \mathrm{~mL} / \mathrm{d}$ for adults or $>400 \mathrm{~mL} / \mathrm{d}$ in children)

VII. In addition, it is required that a documented infection (defined below) is identified

A. Culture-positive infection, or

B. Pathologic tissue source identified, or

C. Clinical response to antimicrobials

\section{Sources of Infection}

Although the leading infective bacterium in burn wounds is Staphylococcus aureus, a recent study showed that the leading causes of death from infection now are multiply resistant organisms, including Pseudomonas and Acinetobacter [2]. The following are the organisms most frequently responsible for infections after burns.

\section{Gram-positive bacteria}

Staphylococcus. Staphylococcus aureus remains the chief cause of burn wound infection [6]. Over recent decades and with the liberal use of broad-spectrum antibiotics, methicillin-resistant $S$. aureus (MRSA) has become the predominant pathogen in the ICU. Colonization with either of these bacteria in an uninjured individual usually is asymptomatic, but they are a source of opportunistic infection that can lead to severe illness and death [7]. Staphylococcus produces toxic byproducts such as proteinases, collagenases, and hyaluronidase that allow it to invade local tissues and disseminate hematogenously, causing generalized systemic infection and sepsis [8]. Methicillin-resistant $S$. aureus now is one of the leading organisms causing invasive infection in burns across the world, with burn units reporting rates of infection greater than $50 \%[9,10]$. In addition to causing 
pneumonia, sepsis, and other sequelae related to invasive burn wound infection, staphylococci are a significant cause of graft loss when the burden of infective organisms exceeds $10^{5}$ CFU [11].

Although vancomycin has been the stand-alone treatment for MRSA infections, over recent years, resistant strains such as vancomycin-intermediate $S$. aureus have been isolated. Other new antimicrobials such as linezolid (an oxazolidinone), tigecycline, daptomycin, quinupristin-dalfopristin, and dalbavancin have been developed specifically to tackle this problem. When using vancomycin for burns, which often involve major changes in fluid status and renal function, maintaining a therapeutic concentration with a trough normally between $10-15 \mathrm{mcg} / \mathrm{mL}$ is imperative. Daily monitoring of vancomycin concentrations enables any needed alterations in dosing.

Streptococcus. Although once the leading cause of burn wound infection, streptococci now are easily eradicated with penicillins. Graft failure is the major complication that is observed occasionally with these bacteria, especially $\beta$ hemolytic streptococci. Of these, Group A (S. pyogenes) is the most problematic, followed by Group B (S. agalactiae) [12].

Enterococcus. The importance of enterococci has been brought to the fore with the emergence of vancomycinresistant Enterococcus (VRE). In a recent review comparing the cause of death from sepsis between consecutive decades (1989-1999 and 1999-2009), a steep decline in the rate of infection with enterococci (25\% to $2 \%$ ) was found [2]. This may be attributable, in part, to the more liberal use of vancomycin during recent years. However, the emergence of VRE should encourage vigilance for this organism, as mortality rates now are greater than that for MRSA (58\% vs. 33\%) [13]. Usually, VRE is treated with linezolid; however, the organism also may be susceptible to a combination of ampicillin and an aminoglycoside or Synercid ${ }^{\circledR}$ (quinupristin-dalfopristin).

\section{Gram-negative bacteria}

Pseudomonas. Pseudomonas is not only the most ubiquitous burn wound pathogen, but also the most likely to be responsible for sepsis leading to burn-linked death $[2,14]$. Both the local environment and the gastrointestinal tract (via translocation of endogenous gastrointestinal flora) are believed to be the main sources of this problematic species of gram-negative bacteria. Pseudomonas aeruginosa is the chief pathogen responsible for respiratory tract infections in the hospital; however, it also may cause invasive burn wound infection because of its preference for moist environments. A superficial wound infection caused by $P$. aeruginosa typically will have a characteristic yellow/green color and a malodorous fruity smell [15]. This may become an invasive infection or ecthyma gangrenosum, causing blue-purplish "punched-out" lesions in the skin. This picture indicates local thrombosis of vessels and requires urgent debridement to remove the newly necrotic tissues.

The treatment of choice for $P$. aeruginosa infection has changed over the years from aminoglycosides to piperacillintazobactam because of developing resistance patterns [16]. The increasing prevalence of multiply drug-resistant $P$. aeruginosa has caused many problems for clinicians looking after the severely burned. This has led to the resurgence of the use of polymyxins, in particular colistin (polymyxin E). Colistin has a substantial side-effect profile, with significant accumulation in important tissues such as the kidney, brain, liver, muscle, and lung (in descending order of frequency) $[17,18]$. Therefore, prolonged use is not recommended, particularly in patients with renal impairment of any kind (the drug is excreted by the kidneys). During administration, patients must be monitored closely for signs of nephrotoxicity and neurotoxicity.

Acinetobacter. Observed with increasing frequency, this organism may lead to numerous opportunistic infections, including pneumonia and those of the surgical site and urinary tract [19]. Second only to $P$. aeruginosa in frequency $[20,21]$, this organism has an enhanced capacity for transfer between patients because of its ability to survive in both dry and wet conditions as well as on both animate and inanimate objects, whether metal or plastic [22]. Although traditionally susceptible to ceftazidime and ciprofloxacin, Acinetobacter has developed resistance to such an extent that only the carbapenems (e.g., imipenem and meropenem) can be relied now on to treat these infections. In cases of pan-resistant Acinetobacter, colistin has become the fallback treatment, as for Pseudomonas infections [23].

Enterobacteriaceae (Escherichia coli, Klebsiella, Enterobacter, Serratia, Proteus). Although less problematic than the organisms already discussed, Enterobacteriaceae are associated with infections in burn patients, most often causing pneumonia and urinary tract infections [24]. Although these pathogens have greater sensitivity to antibiotics than do other gram-negative organisms, resistance patterns have been changing over recent years such that carbapenems and fourthgeneration cephalosporins are used increasingly for problematic infections because of the new sensitivity patterns [25].

Anaerobes. Anaerobic bacteria now are rarely a cause of invasive infection in burns. The most common anaerobic organisms isolated are the Bacteroides and Fusobacterium spp. These are sometimes observed in myonecrosis secondary to electrical or crush injury when devascularized muscle is retained in a compartment. In these cases, broad-spectrum antibiotics should be given until sensitivities are available so that an appropriate drug can be given [26,27].

Fungi/yeasts. Colonization with fungi has become an increasing problem because the introduction of topical antimicrobials and liberal use of broad-spectrum antibiotics. This has resulted in a surge in invasive fungal infection, which has been linked to higher death rates regardless of the extent of the burn, coincident inhalation injury, or patient age [28]. In a recent review of 15 burn units, fungi were isolated at least once from $6.3 \%$ of 6,918 patients [29], with positive cultures being obtained most commonly from the wound itself followed by (in order of decreasing frequency) respiratory, urine, and blood specimens.

Candida albicans is the fourth most frequently found pathogen in blood cultures from ICU patients [30]. However, invasive infection with molds such as Aspergillus is correlated more closely with death [30]. Non-albicans Candida spp. are becoming an increasingly frequent cause of invasive 
candidiasis, with their incumbent resistance to conventional antifungal agents such as fluconazole [31]. Recent guidelines list caspofungin (an echinocandin) as the treatment of choice in these cases [32]. The trend away from $C$. albicans also is worrying, as deep fungal infection associated with a higher mortality rate is associated with $C$. tropicalis and $C$. kruse $i$ [33,34]. Aspergillus, Fusarium, and zygomycetes (Rhizopus and Mucor) are isolated less often but have a far greater propensity for aggressive, invasive infection than do yeasts [35].

The most successful treatment for fungal infection is prevention via swift removal of all burned tissue and closure of wounds with autografts. In the presence of active noncandidal infection, voriconazole is the first-line treatment followed by amphotericin B (lipid formulation). An echinocandin such as caspofungin can be considered for combination treatment of Aspergillus and Fusarium [23].

Viruses. Primary viral burn wound infection, although rare, certainly is possible. The most common cause is reactivation of latent infection because of the debilitated immunosuppressed state of the patient after a substantial injury. Herpes viruses, especially herpes simplex and varicellazoster, have by far the greatest occurrence. The viral infections arising most frequently in healing burn wounds are caused by herpes simplex virus. These also can occur in donor sites. Typical patterns of vesicles are found at the edge of the wound, and these vesicles coalesce into a confluent raw area. Prompt treatment with both oral and topical antiviral therapies such as acyclovir should be continued for a minimum of 10 days.

\section{Special considerations}

In addition to obvious sources and areas of infection, there are other vulnerable tissues and organ systems in the burn patient that require special consideration. Those associated with indwelling catheters such as intravenous and intraarterial lines and urinary catheters are paramount. These will be discussed, along with the respiratory and gastrointestinal systems.

Intravenous and intra-arterial catheters. Intravenous and intra-arterial catheters provide access to the blood, an essential route for delivery of resuscitative and pharmacologic therapy, which are crucial for treating burn patients. Regardless of aseptic technique practice, rates of catheterassociated infection and septic thrombophlebitis in the burn patient are as high as 57\% [36-38].

Franchesi et al. [39] described a 50\% correlation between the organisms cultured from the tip and the connectors of the catheter within $2 \mathrm{~d}$ of placement, and they found that a negative correlation existed between the frequency of catheter infection and the distance separating the point of catheter insertion and the burn wound. These data support the hypothesis that catheter infections arise primarily from burn wound contamination migrating to the catheter tip.

Serious complications often can be avoided by following strict aseptic techniques. General guidelines for protecting against catheter-related infections are as follows [40]:

1. Training healthcare staff on the correct procedures for inserting and maintaining catheters, including indications and suitable infection control practices;
2. Use of a mask, hat, gown, and sterile gloves and drapes during placement of central venous catheters (CVCs);

3. Use of $2 \%$ chlorhexidine to prevent infection;

4. Avoiding routine CVC changes;

5. In the event that infection continues while adhering to guidelines 1-3, use short-term CVCs coated with an antibiotic, antiseptic, or both.

Serial positive blood cultures without a sign of local infection should alert clinicians to suspect suppurative thrombophlebitis. In this case, it is necessary to remove the catheter promptly, and, if the diagnosis is confirmed, to perform urgent operative excision of the cannulated vessel to avoid progressive sepsis.

Genitourinary system. Hematogenous dissemination of bacteria and fungi can lead to urinary tract infections in the susceptible burn patient, but these organisms often are introduced via prolonged or unnecessary use of urinary catheters. If catheters are used under strict guidelines (e.g., aseptic techniques, catheter care, routine urine monitoring, and early removal), urinary tract infections can be avoided. When infection does occur, patients should be treated with appropriate systemic antibiotics and antifungal agents.

Respiratory tract. Respiratory tract infections are a major trigger of septic deaths after burns, second only to infection of burn wounds leading to septicemia. Pneumonia has two basic etiologies: Organisms can enter either through direct contamination of the airway or hematogenously. The organisms cultured usually are reflective of burn wound flora. The diagnosis of pneumonia is a clinical one and requires a chest radiograph showing two of the following: A new and enduring infiltrate, consolidation, or capitation [13]. In burn patients, sepsis is defined as a clinical deterioration in status and a change or purulence in the sputum. In the mechanically ventilated burn patient, close attention should be paid to ventilator-associated pneumonia, which is pneumonia appearing greater than two $\mathrm{d}$ after intubation in patients who showed no signs of pneumonia before this time.

Pneumonia should be diagnosed on the basis of the clinical picture in combination with two of these three criteria: A radiograph of the chest showing a new, persistent infiltrate, consolidation, or capitation; sepsis (as defined for burns); or a change or purulence in expectorated or aspirated sputum [1]. Sputum examination should include observations of the color, amount, consistency, odor, and gram staining.

Gastrointestinal system. Placement of a central venous catheter may be necessary for proper alimentary support. Placement through the burned tissue should be avoided. If unavoidable, the area should be cleansed daily and topical antibiotics applied. The catheter should be cultured routinely to identify any micro-organisms gaining entry via the surgical puncture wound.

The gastrointestinal tract also can serve as the chief source of infection after burn injury. In large burns, the abdomen often is involved, and this can lead to the perception of limitation during the clinical examination. The affected skin should not be allowed to interfere with a proper physical examination. Burn patients, like all patients, can suffer from, 
and should be evaluated for, appendicitis, intussusception, and bowel obstruction. Intra-peritoneal dialysis may be necessary to aid compromised renal function and may also serve as a source of infection.

Clostridium difficile is a well-known opportunistic pathogen in severely ill individuals with a history of antibiotic use and in immunocompromised patients. Surveillance for the organism remains necessary, although its clinical observance may wane in the wake of the emergence of methicillinresistant Staphylococcus and the use of intravenous vancomycin.

Burn patients also should be evaluated for necrotizing enterocolitis, with the classic presentation of high-spiking fevers accompanied by left upper-quadrant pain, referred pain in the left shoulder from the adjacent diaphragm, splenic hypertrophy, and tenderness. Diagnostic imaging should be performed, and broad-spectrum antibiotics with activity against streptococci and both aerobic and anaerobic gramnegative bacilli should be administered on confirmation of the infection.

\section{Surveillance, sampling, and prevention}

Burn wound cultures. Identifying and treating infections after burn injury has relied routinely on clinical impression and qualitative findings from surface swabs, although analysis of burn wound biopsies through quantitative bacteriology is needed to confirm burn wound infection and improves patient management and outcomes [41].

Historically, quantitative wound cultures have been utilized to monitor healing. Culturing the surface of burn wounds can help in determining whether pathogens are present; however, the results do not indicate wound status. It has been reported that death after burn injury correlates with the presence of $>10^{5}$ organisms/g of tissue or histologic signs of invasive infection [42].

Histologic analysis of burn wound biopsies is the only means by which microbial invasion can be identified definitively and invasive disease can be diagnosed. Quantitative culture is inadequate for detecting invasive infection. Therefore, histologic determinations of invasive infection should be paired with non-quantitative culture to identity pathogens and determine their sensitivity to antimicrobial agents. This approach will aid in diagnosis and guide choices for suitable interventions. One should keep the following points in mind:

- Early cultures should be negative or have low counts of sensitive gram-positive organisms. Positive cultures or high counts suggest early contamination of the burn wound.

- If invasive burn wound infection is suspected, wound culture and histologic analysis can aid in confirmation of the diagnosis.

- Routine culturing and identification of colonization may aid in empiric antimicrobial agent coverage if the patient subsequently becomes ill.

- Increasing colony counts may indicate a need to change topical antimicrobial agents.

- Not all organisms are created equal. Wound colonization with particularly virulent or resistant organisms may be a predictor of impending invasive burn wound infection.
- Operative wound colony counts $>10^{6}$ suggest a high risk of infectious complications and graft failure.

- Burn wound culture results may aid in the evaluation of nosocomial spread of organisms and guide infection control practice.

- Regular catheter changes-every 5 to $6 \mathrm{~d}$ for central catheters-can decrease the likelihood of catheterrelated infections. The Nosocomial Infection Surveillance System of the U.S. Centers for Disease Control and Prevention has shown that CVC-derived primary infections of the blood stream occur at elevated rates in burn ICUs.

\section{Summary}

Developments in critical care and surgical approaches to treating burn wounds together with antimicrobial treatments have reduced significantly the morbidity and mortality rates associated with this injury. However, several resistant organisms have emerged as the maleficent cause of invasive infection, including MRSA, VRE, Pseudomonas, Acinetobacter, non-albicans Candida species, and Aspergillus. Advances in antimicrobial therapies and the release of new classes of antibiotics have certainly added to the armamentarium of resources for the clinician. Nevertheless, strict infection control measures, constant wound surveillance with regular sampling of tissues for quantitative culture, and early excision and wound closure remain the principal adjuncts to control invasive infection in burn patients.

\section{Study Support and Author Disclosure Statement}

This study was supported by the National Institutes of Health (R01-GM087285-01,P50-GM060338, R01-GM055687, and T32-GM8256), the National Institute on Disability and Rehabilitation Research (H133A070026 andH133A70019), the CFI Leader's Opportunity Fund (Project \#25407), Canadian Institutes of Health Research (grant No. 123336), the Physicians' Services Incorporated Foundation-Health Research Grant Program, and Shriners Hospitals for Children (grants 71001, 84080, 71008, and 79135). CCF is an ITS Career Development Scholar supported, in part, by NIH KL2RR029875 and NIH UL1RR029876. The authors have no conflicts of interest to declare.

\section{References}

1. Greenhalgh DG, Saffle JR, Holmes JHT, et al. American Burn Association consensus conference to define sepsis and infection in burns. J Burn Care Res 2007;28:776-790.

2. Williams FN, Herndon DN, Hawkins HK, et al. The leading causes of death after burn injury in a single pediatric burn center. Crit Care 2009;13:R183.

3. American Burn Association Association. Burn incidence and treatment in the US: 2012 Facts sheet. Chicago. The Association, 2012.

4. American Burn Association. 2012 National Burn Repository, 2012. Chicago. The Association, 2012.

5. Barret JP, Herndon DN. Effects of burn wound excision on bacterial colonization and invasion. Plast Reconstr Surg 2003;111:744-750. 
6. Edwards-Jones V, Greenwood JE. What's new in burn microbiology? James Laing Memorial Prize Essay 2000. Burns 2003;29:15-24.

7. Cook N. Methicillin-resistant Staphylococcus aureus versus the burn patient. Burns 1998;24:91-98.

8. de Macedo JL, Rosa SC, Castro C. Sepsis in burned patients. Revis Socied Bras Med Trop 2003;36:647-652.

9. Prasanna M, Thomas C. A profile of methicillin-resistant Staphylococcus aureus infection in the burn center of the Sultanate of Oman. Burns 1998;24:631-636.

10. Thabet L, Turki A, Ben Redjeb S, Messadi A. [Bacteriological profile and antibiotic resistance of bacterial isolates in a burn department] (Fre). Tun Med 2008;86: 1051-1054.

11. Robson MC, Krizek TJ, Heggers JP. Biology of surgical infection. In: Ravitch MM, ed. Current Problems in Surgery. Chicago. Year Book Medical Publishers, 1973:1-62.

12. Wilson GR, French GW, Sully L. Loss of split thickness skin grafts due to non-group A beta-haemolytic streptococci. Ann R Coll Surg Engl 1988;70:217-219.

13. von Baum H, Ober JF, Wendt C, et al. Antibiotic-resistant bloodstream infections in hospitalized patients: Specific risk factors in a high-risk population? Infection 2005;33: 320-326.

14. Mayhall CG. The epidemiology of burn wound infections: Then and now. Clin Infect Dis 2003;37:543-550.

15. McManus AT, Mason AD Jr, McManus WF, Pruitt BA Jr. Twenty-five year review of Pseudomonas aeruginosa bacteremia in a burn center. Eur J Clin Microbiol 1985;4:219223.

16. Walton MA, Villarreal C, Herndon DN, Heggers JP. The use of aztreonam as an alternate therapy for multi-resistant Pseudomonas aeruginosa. Burns 1997;23:225-227.

17. Kunin CM, Bugg A. Binding of polymyxin antibiotics to tissues: The major determinant of distribution and persistence in the body. J Infect Dis 1971;124:394-400.

18. Kunin CM, Bugg A. Recovery of tissue bound polymyxin B and colistimethate. Proc Soc Exp Biol Med 1971;137: 786-790.

19. Zarrilli R, Crispino M, Bagattini M, et al. Molecular epidemiology of sequential outbreaks of Acinetobacter baumannii in an intensive care unit shows the emergence of carbapenem resistance. J Clin Microbiol 2004;42:946-953.

20. Chim H, Tan BH, Song C. Five-year review of infections in a burn intensive care unit: High incidence of Acinetobacter baumannii in a tropical climate. Burns 2007;33:1008-1014.

21. Keen EF 3rd, Robinson BJ, Hospenthal DR, et al. Prevalence of multidrug-resistant organisms recovered at a military burn center. Burns 2010;36:819-825.

22. Corbella X, Montero A, Pujol M, et al. Emergence and rapid spread of carbapenem resistance during a large and sustained hospital outbreak of multiresistant Acinetobacter baumannii. J Clin Microbiol 2000;38:4086-4095.

23. Branski LK, Al-Mousawi A, Rivero H, et al. Emerging infections in burns. Surg Infect 2009;10:389-397.

24. Patel JA, Williams-Bouyer N. Infections in burn patients. In: Feigin RD, ed. Feigin \& Cherry's Textbook of Pediatric Infectious Diseases. Philadelphia. Saunders/Elsevier, 2009: 1139-1151.

25. Guggenheim M, Zbinden R, Handschin AE, et al. Changes in bacterial isolates from burn wounds and their antibiograms: A 20-year study (1986-2005). Burns 2009;35: 553-560.
26. Church D, Elsayed S, Reid O, et al. Burn wound infections. Clin Microbiol Rev 2006;19:403-434.

27. Murray PM, Finegold SM. Anaerobes in burn-wound infections. Rev Infect Dis 1984;6(Suppl 1):S184-S186.

28. Horvath EE, Murray CK, Vaughan GM, et al. Fungal wound infection (not colonization) is independently associated with mortality in burn patients. Ann Surg 2007; 245:978-985.

29. Ballard J, Edelman L, Saffle J, et al. Positive fungal cultures in burn patients: A multicenter review. J Burn Care Res 2008;29:213-221.

30. Wisplinghoff H, Bischoff T, Tallent SM, et al. Nosocomial bloodstream infections in US hospitals: Analysis of 24,179 cases from a prospective nationwide surveillance study. Clin Infect Dis 2004;39:309-317.

31. Leroy O, Mira JP, Montravers P, et al. Comparison of albicans vs. non-albicans candidemia in French intensive care units. Crit Care 2010;14:R98.

32. Pappas PG, Kauffman CA, Andes D, et al. Clinical practice guidelines for the management of candidiasis: 2009 Update by the Infectious Diseases Society of America. Clin Infect Dis 2009;48:503-535.

33. Alangaden GJ. Nosocomial fungal infections: Epidemiology, infection control, and prevention. Infect Dis Clin North Am 2011;25:201-225.

34. Hope W, Morton A, Eisen DP. Increase in prevalence of nosocomial non-Candida albicans candidaemia and the association of Candida krusei with fluconazole use. J Hosp Infect 2002;50:56-65.

35. Becker WK, Cioffi WG Jr, McManus AT, et al. Fungal burn wound infection: A 10-year experience. Arch Surg 1991;126:44-48.

36. Samsoondar W, Freeman JB, Coultish I, Oxley C. Colonization of intravascular catheters in the intensive care unit. Am J Surg 1985;149:730-732.

37. Maki DG, Jarrett F, Sarafin HW. A semiquantitative culture method for identification of catheter-related infection in the burn patient. J Surg Res 1977;22:513-520.

38. O`Neill JA Jr, Pruitt BA Jr, Foley FD, Moncrief JA. Suppurative thrombophlebitis: A lethal complication of intravenous therapy. J Trauma 1968;8:256-267.

39. Franeschi D, Gerding RL, Phillips G, Fratianne RB. Risk factors associated with intravascular catheter infections in burned patients: A prospective, randomized study. J Trauma Acute Care Surg 1989;29:811-815.

40. O'Grady NP, Alexander M, Dellinger EP, et al. Guidelines for the prevention of intravascular catheter-related infections. Morbid Mortal Wkly Rep Recommend Rep 2002;51: $1-29$.

41. Pruitt BA Jr, McManus AT. The changing epidemiology of infection in burn patients. World J Surg 1992;16:57-67.

42. Pruitt BA Jr, McManus AT, Kim SH, Goodwin CW. Burn wound infections: Current status. World J Surg 1998;22: 135-145.

Address correspondence to: Dr. Celeste C. Finnerty Shriners Hospitals for Children 815 Market Street Galveston, TX 77550

E-mail: ccfinner@utmb.edu 